\title{
Severe high grade stenosing hyperkeratosis of the esophagus after ingestion of alkali: successful treatment by argon plasma coagulation
}

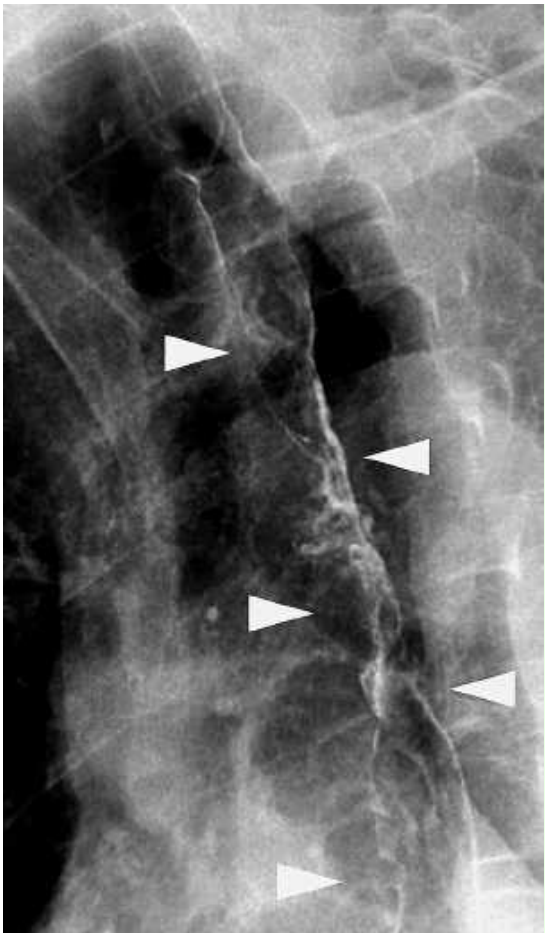

Fig. 1 Esophagography with water-soluble iodine contrast agent, in left anterior oblique position, showed irregular mucosa of length approximately $9 \mathrm{~cm}$ (white arrowheads) in the mid esophagus, and high grade stenosis.

The ingestion of acids or lyes may lead to reversible or irreversible lesions of the upper gastrointestinal tract [1]. In cases of alkali ingestion, esophageal strictures concomitant to dysphagia constitute a major problem of the long-term course. Regular follow-up by endoscopy is recommended owing to the significantly increased risk of developing esophageal carcinoma [2].

In August 2005, a 71-year-old patient with serious dysphagia and known scarred esophageal stricture subsequent to ingestion of alkali presented for further diagnosis and treatment. Esophagography ( $\odot$ Fig. 1) showed an irregular luminal constriction.

During the initial esophagogastroscopy the stenosis $(23-32 \mathrm{~cm}$ from the incisors) proved impassable for the endoscope (external diameter $5.9 \mathrm{~mm}$, GIFXP160; Olympus, Hamburg, Germany) (॰ Fig. 2).

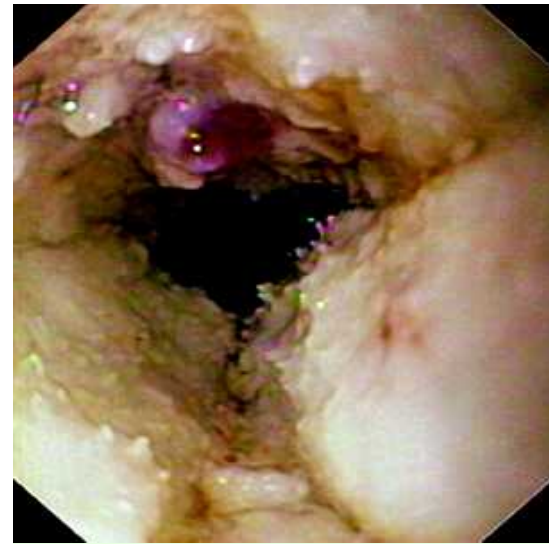

Fig. 2 Initial esophagogastroscopy: the esophageal stenosis ( $23-32 \mathrm{~cm}$ from the dental arch) was impassable for the GIF-XP160 endoscope (external diameter $5.9 \mathrm{~mm}$ ).

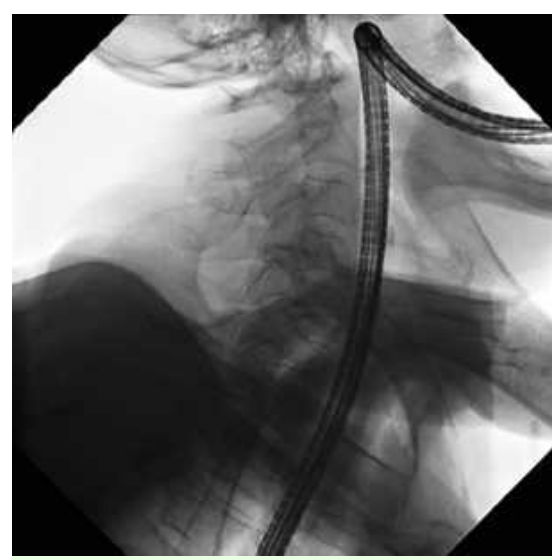

Fig. 3 Bougienage proved ineffective as the bougie, similarly to the endoscope imaged here, bent due to the high resistance within the stenosis.

Bougienage followed by balloon dilation was done over several sessions. However bougienage proved to be ineffective as the bougie bent due to the high resistance within the stenosis ( $\bullet$ Fig. 3).

Despite these therapeutic approaches no positive clinical results could be achieved. After neoplasia had been ruled out, argon plasma coagulation (APC) was done in repeated sessions to ablate the circular hyperkeratoses ( $\bullet$ Fig. 4), using a high frequency device (40-60 W, 0.5-0.61/min; BOWA, Gomaringen, Germany).

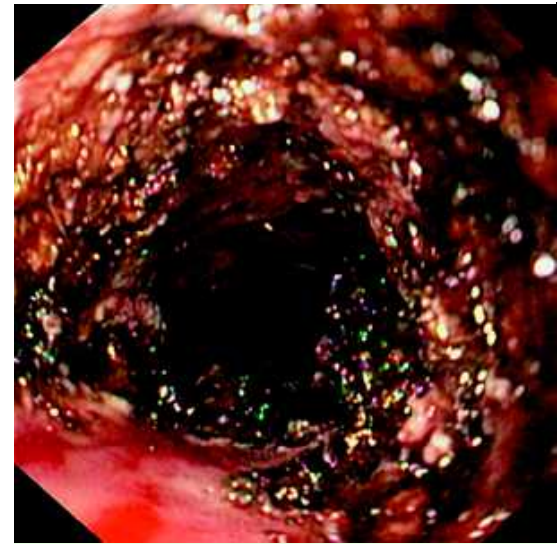

Fig. 4 Argon plasma coagulation (APC) was done (high frequency device, $40-60 \mathrm{~W}$, $0.5-0.61 / \mathrm{min}$; BOWA) to ablate the circular hyperkeratoses.

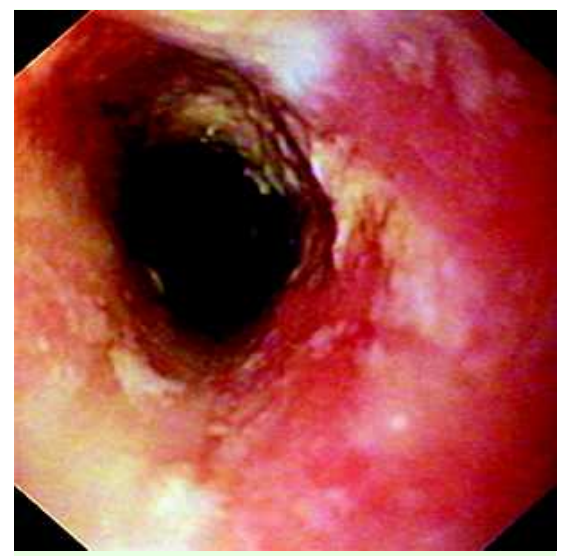

Fig. 5 After APC treatment had been completed, the standard GIF-Q160 endoscope (external diameter $9.8 \mathrm{~mm}$ ) could be passed without difficulty.

At the end of this treatment ( $\bullet$ Fig. 5), passage of the GIF-Q160 endoscope (9.8 $\mathrm{mm}$ ) presented no problems.

Initially an esophageal carcinoma had been suspected, but in multiple tissue biopsies the histopathological examination revealed parts of a distinctively hyperkeratotic squamous epithelium with basal cell proliferation without evidence of malignancy ( Fig. 6).

Using APC, successful desquamation of the hyperkeratotic areas, with re-epithelialization after thermal destruction of the tissue, was achieved. After comple- 


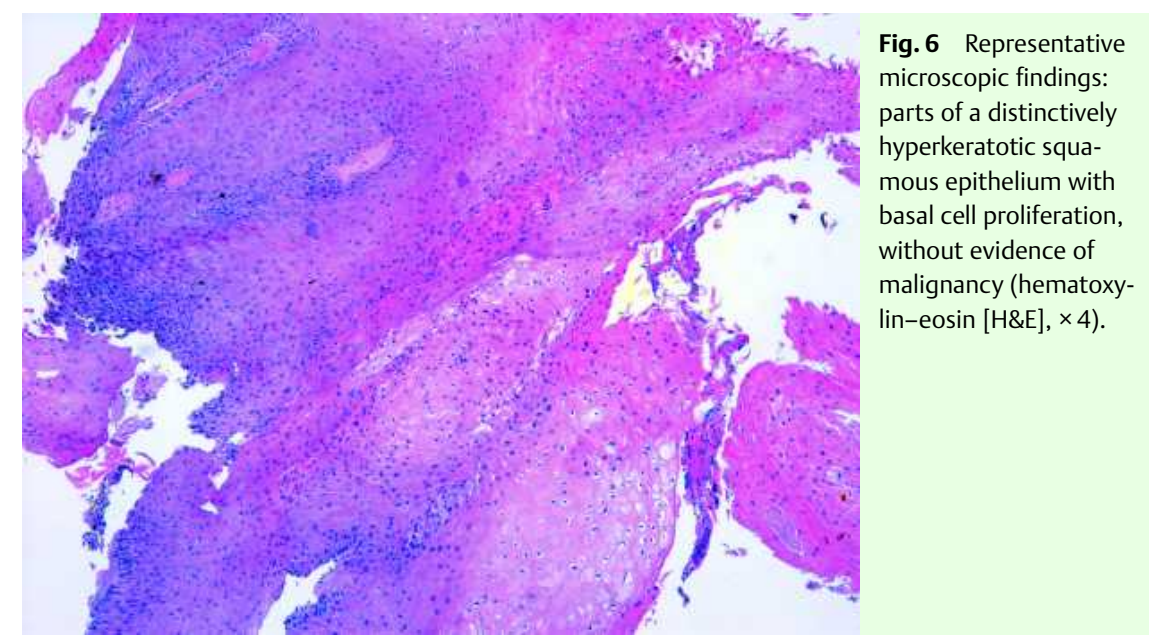

tion of the treatment in October 2006, ingestion of liquid and solid food was again possible and the patient had a noteworthy increase in weight.

In our patient, APC proved to be an effective and safe procedure for treating an esophageal stenosis with distinctive hyperkeratotic changes. Using this therapeutic approach, stent implantation or a surgical procedure was avoided.

Endoscopy_UCTN_Code_CCL_1AB_2AC_3AD

Endoscopy_UCTN_Code_TTT_1AO_2AD

\section{References}

1 Ertekin C, Alimoglu O, Akyildiz H et al. The results of caustic ingestions. Hepatogastroenterology 2004; 51: 1397-1400

2 Obermaier R, Hopt UT, Fischer A. Accidental and suicidal caustic gastric ingestion. Viszeralchirurgie 2007; 42: 14-21

Bibliography

DOI $10.1055 / \mathrm{s}-2008-1077643$

Endoscopy 2008; 40: E260-E261

(c) Georg Thieme Verlag KG Stuttgart · New York . ISSN 0013-726X

Corresponding author

\section{F. Boxberger, MD}

Medizinische Klinik 1 ,

Universitätsklinikum Erlangen

Ulmenweg 18

91054 Erlangen

Germany

Fax: +49-91-318535062

Frank.Boxberger@uk-erlangen.de 